\title{
Prohibitin as a novel autoantigen in rheumatoid arthritis
}

\author{
LI-LI SHI ${ }^{1 *}$, PENG CHEN ${ }^{1 *}$, YI-PING XUN ${ }^{l}$,WEI-KANG YANG ${ }^{l}, C H U N-H E Y A N G^{l}$, \\ GUANG-YU CHEN ${ }^{2}, H O N G-W U D U^{l}$
}

${ }^{1}$ School of Chemistry \& Biotechnology Engineering, University of Science \& Technology Beijing, Beijing, China ${ }^{2}$ ImmunoHunt Corporation, Beijing, China

*Equal contributors.

\begin{abstract}
The aim of this study was to verify whether prohibitin is a novel autoantigen in rheumatoid arthritis.

Material and methods: First, recombinant human prohibitin (rhPHB) protein was cloned, expressed, and purified. Then the anti-prohibitin autoantibodies were detected by western blotting by using rhPHB protein to incubate sera from patients with rheumatoid arthritis (RA). Next, immunoprecipitation was employed to further illustrate whether anti-prohibitin antibodies exist in RA patients. And finally, autoantibodies against the rhPHB protein were investigated using a homemade ELISA kit through the assessment of 258 real clinical samples.

Results: It was revealed that anti-prohibitin antibodies existed in the sera of patients with RA. Reactivity of serum IgG against rhPHB was detected in 26 of 86 RA patients (30.3\%), 7 of 86 systemic lupus erythematosus (SLE) patients (8.1\%), and 1 of 86 apparently healthy donors (HC) (1.2\%).

Conclusions: Prohibitin was proved to be a novel autoantigen and the corresponding anti-prohibitin autoantibodies were present in the RA patients' blood circulation.
\end{abstract}

Key words: autoantibodies, autoantigen, autoimmune disease, prohibitin, rheumatoid arthritis.

(Centr Eur J Immunol 2015; 40 (1): 78-82)

\section{Introduction}

Rheumatoid arthritis (RA) is a chronic systemic autoimmune disease, which is characterised by chronic inflammation of synovial joints, eventually results in erosion damage in articular cartilage and bone, joint swelling, deformity, and dysfunction $[1,2]$. Although the aetiology of RA remains unclear, autoimmunity is believed to play a pivotal role in the pathological process of RA [3, 4]. An increasingly diverse set of autoantigens, including rheumatoid factor [5], filaggrin [6], vimentin [7], $\alpha$-enolase [8], collagen type II [9], calpastatin [10], translation initiation factor 4G1 [11], and YKL-39 [12], have been found over the past years, which can trigger the immune response; they give useful information and clues as to the aetiology of RA [13, 14].

Prohibitin was believed to regulate human inflammation [15] and be involved against oxidative stress in the intestinal epithelial cells [16]. Also, prohibitin participates in regulating the expression of some cytokines [17], such as interleukin (IL)-6, which can promote the pathogenesis of RA [18]. Tumor necrosis factor $\alpha$ (TNF- $\alpha)$ is a proinflammatory cytokine, which plays an important role in the pathogenesis of RA [19]. Prohibitin can also inhibit TNF $\alpha$ induced $N F-\kappa B$ activation [20]. In our previous study [21], prohibitin was suggested as a novel autoantigen of Behçet's disease. Here, we further investigate whether prohibitin is a novel autoantigen in rheumatoid arthritis, which may help to illuminate whether prohibitin can play a role in the process of RA.

\section{Material and methods}

\section{Sample collection}

Serum samples from 86 patients with RA were collected, including 75 female and 11 male, with ages ranging from 18 to 74 years (average age 41 years). All the patients conformed to the criteria proposed by the 1987 American Rheumatism Association for the classification of RA [22]. Sera was taken from 86 patients with systemic lupus erythematosus (SLE) who were diagnosed according to the defined criteria [23], and sera from 86 healthy volunteers (HC) were used as a control. Initially, samples from eight RA patients were collected in June 2013. The other samples were collected from September 2013 to June 2014

Correspondence: Prof. Hongwu Du, University of Science and Technology, 30 Xueyuan Road, Haidian District, 100083 Beijing, China, e-mail: hwdu93@126.com 
for a large-scale test using the ELISA method. All of the patients involved in the study were treated at the Chinese People's Liberation Army General Hospital. This study was approved by the Ethical Committee of the Chinese People's Liberation Army General Hospital, and each patient involved in this study gave informed consent. The samples were collected, dispensed, and stored at $-80^{\circ} \mathrm{C}$ for further testing.

\section{Expression and purification of prohibitin}

The procedure of protein expression and purification was basically performed as before [24]. In brief, total RNA was isolated using TRIzol reagent (Invitrogen, Carlsbad, CA), based on the acid guanidinium thiocyanate-phenol-chloroform extraction method. Reverse transcription-polymerase chain reaction (RT-PCR) was performed according to the manufacturer's instruction (Fermentas, Glen Burnie, MD). Primers were designed according to the coding sequences for human PHB, and EcoR I and Hind III restriction sites were placed at their respective terminal ends to facilitate sub-cloning of the PCR product into the expression vector pET-28a(+). Protein was overexpressed in the Escherichia coli BL21 (DE), followed by the purification of recombinant proteins using Ni-NTA resin (Qiagen, Hilden, Germany). The concentrations of proteins were determined by BCA kit (Boisynthesis Biotechnology, Beijing, China). Purified recombinant protein was stored at $-80^{\circ} \mathrm{C}$.

\section{Western blotting}

Western blotting was performed as described elsewhere [25]. The rhPHB protein was loaded on $12 \%$ SDSPAGE ( $4 \mu \mathrm{g}$ total protein per well). Then the proteins on the gel were transferred onto PVDF membrane (Merck Millipore, MA), which was then incubated with serum samples from eight patients with RA (the first batch of samples collected in the lab) or serum samples from eight patients with HC. Sera were diluted 1 : 500. Bound antibodies were detected by incubating with horseradish peroxidase-conjugated goat anti-human IgG (ImmunoHunt, Beijing, China). Each stripe was followed by three washes with PBS/Tween $0.05 \%$ (PBST) buffer. The positive bands were detected using enhanced chemiluminescence kit (Applygen, Beijing, China) on the basis of the instructions of the manufacture.

\section{Immunoprecipitation}

The rhPHB protein $(5 \mu \mathrm{g})$ was incubated with RA sera (equal volumes from three positive RA patients in western blotting detection) overnight at $4{ }^{\circ} \mathrm{C}$ on a rotator. Subsequently, $50 \mu \mathrm{l}$ of protein A-Sepharose beads (Sigma, MO) washed with PBS were added and incubated for 4 hours at $4^{\circ} \mathrm{C}$. The immunoprecipitates were washed three times in $200 \mu \mathrm{l}$ PBS. Then the immunoprecipitates and supernatant were suspended in a sample loading buffer and analysed by $12 \%$ SDS-PAGE.

\section{Mass spectrometry}

The target protein in the immunoprecipitates was excised with the gel and washed with $50 \%$ acetonitrile until destaining, and the gel was dehydrated. $10 \mathrm{mM}$ dithiothreitol (DTT) was added to cover the gel, and it was incubated at $37^{\circ} \mathrm{C}$ for two hours. After cooling to room temperature, the DTT solution was replaced by the same volume of $25 \mathrm{mM} \mathrm{NH}_{4} \mathrm{HCO}_{3}$ containing $55 \mathrm{mM}$ iodoacetamide and incubated for 45 minutes in the dark. Alkylated gel was dried in a speed vac concentrator (Savant Instruments, NY) and digested with trypsin (Sigma, MO). Finally, the peptide fragments were analysed by LC-MALDI-TOF/TOF of AB5800 Proteomics Analyser (Applied Biosystems, MA). Mass spectrometric data were analysed by Mascot bioinformatics database (Matrix Sciences, London, UK).

\section{ELISA}

ELISA with rhPHB protein was performed as described previously [25]. Briefly, $0.1 \mu \mathrm{g} / \mathrm{ml}$ of rhPHB protein was coupled covalently to 96-well microtitre plates (Corning, NY). The plates were then blocked with $200 \mu$ of $5 \%$ goat serum for 2 hours at $37^{\circ} \mathrm{C} .100 \mu \mathrm{l}$ of sera at $1: 100$ dilution was added and incubated for 2 hours at $37^{\circ} \mathrm{C}$. Plates were then washed five times with $0.1 \%$ PBST. Then $100 \mu$ of horseradish peroxidase-conjugated mouse anti-human IgG diluted at $1: 15,000$ was added. Bound antibodies were detected with tetramethylbenzidine (TMB) A ( $0.1 \mathrm{M}$ citric acid, $0.2 \mathrm{M} \mathrm{Na}_{2} \mathrm{HPO}_{4}, 0.6 \mathrm{~g}$ hydroperite/l) and TMB B ( $5 \mathrm{mM}$ citric acid, $0.4 \mathrm{mM}$ EDTA-Na ${ }_{2}$, and $0.2 \mathrm{~g} \mathrm{TMB} / \mathrm{l}$ ) as substrate. Finally, the reaction was stopped by adding $2 \mathrm{M} \mathrm{H}_{2} \mathrm{SO}_{4}$. The absorbance at $450 \mathrm{~nm}$ was measured with a microplate reader (Tecan, Hombrechtikon, Switzerland).

\section{Statistical analysis}

Mean, SD, and $t$-test were evaluated by SPSS software (Version 17, Chicago, IL), and the result was considered statistically significant if $p<0.05$. The threshold to define a positive result was a value higher than that of the healthy controls (Mean + 3 SD).

\section{Results}

\section{Cloning and expression of prohibitin}

The PCR products showed a single band at the position of 819 bp as indicated on $1 \%$ agarose gel (Fig. 1A). The extracted band was excised by EcoR I and Hind III and then ligated into the pET-28a (+) vector, which had been digested with EcoR I and Hind III, to form the recombinant plasmid. The construct was transformed in E. coli BL21 (DE). After six hours of induction by isopropyl- $\beta$-D-thio- 

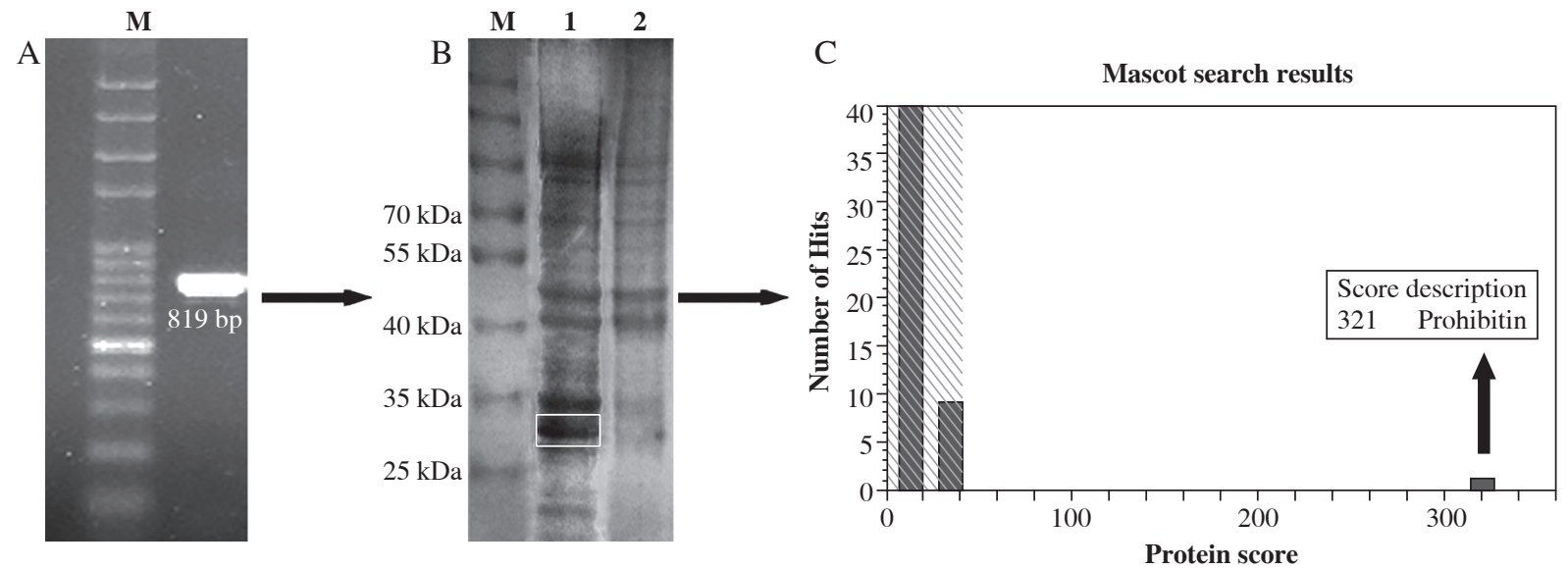

Fig. 1. Expression of prohibitin. A) PCR of the prohibitin gene. Agarose gels showing the amplified 819-bp fragment. B) Sodium dodecyl sulphate-poly-acrylamide gel electrophoresis (SDS-PAGE) analysis of prohibitin protein. M, protein marker; lane 1, cell extracts of pET-28a(+)-prohibitin/BL21 after isopropyl- $\beta$-D-thiogalactopyranoside (IPTG) induction for $6 \mathrm{~h}$ at $37^{\circ} \mathrm{C}$; lane 2, cell extracts of pET28a (+)/BL21 after IPTG induction. C) Prohibitin was identified by mass spectrometry

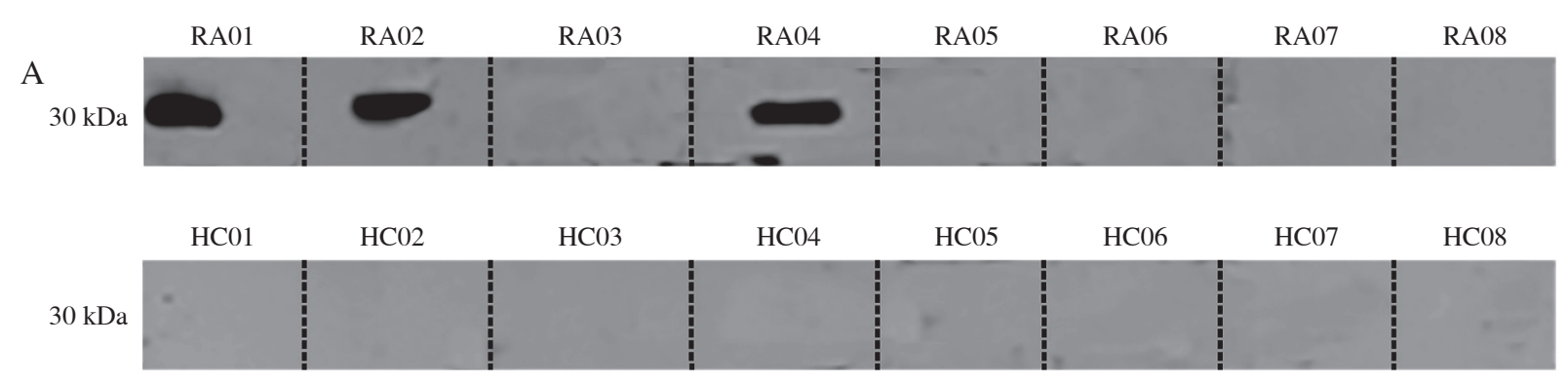

RA - sample from rheumatoid arthritis, $\mathrm{HC}$ - sample from healthy controls
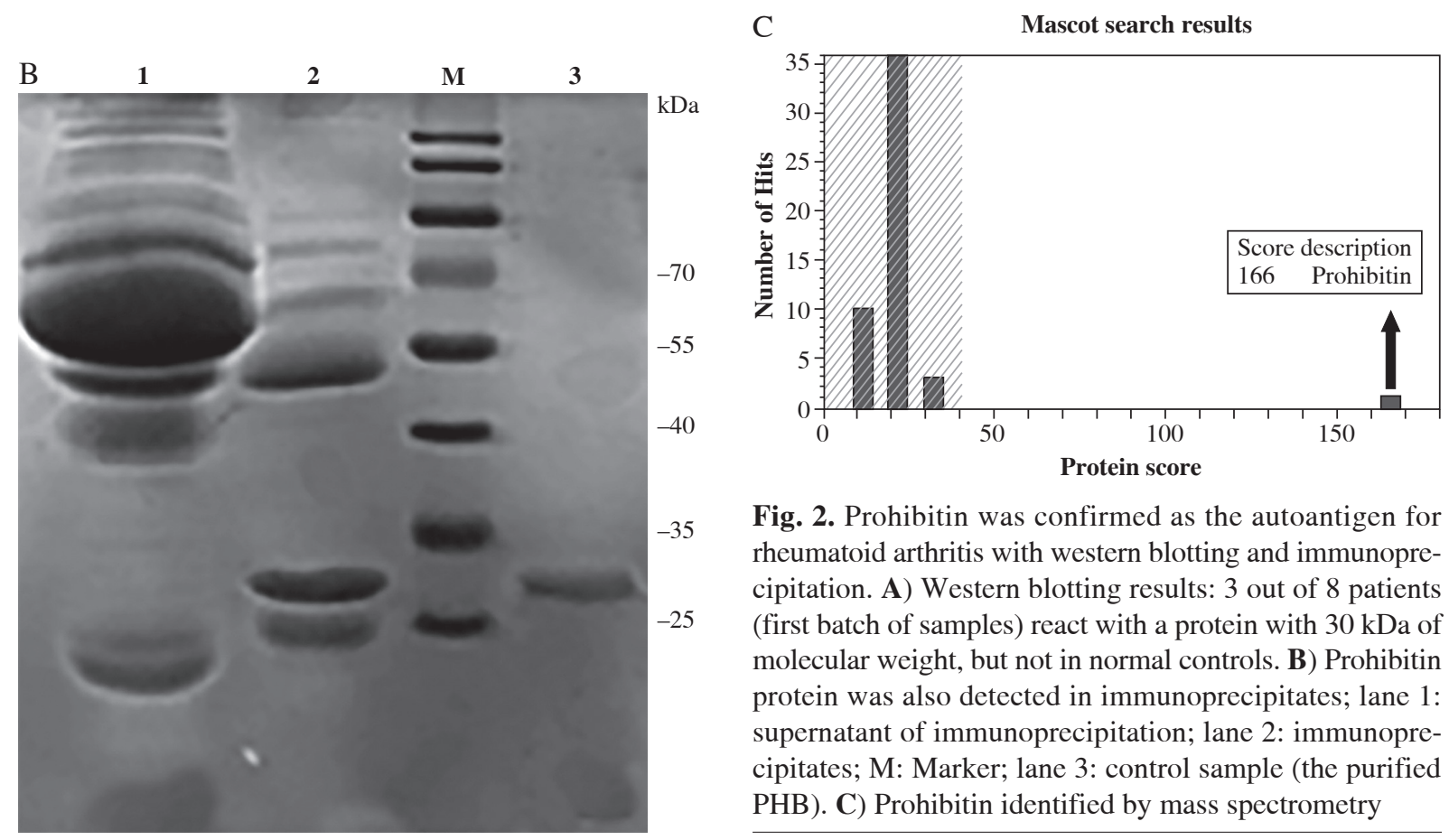

Fig. 2. Prohibitin was confirmed as the autoantigen for rheumatoid arthritis with western blotting and immunoprecipitation. A) Western blotting results: 3 out of 8 patients (first batch of samples) react with a protein with $30 \mathrm{kDa}$ of molecular weight, but not in normal controls. B) Prohibitin protein was also detected in immunoprecipitates; lane 1: supernatant of immunoprecipitation; lane 2: immunoprecipitates; M: Marker; lane 3: control sample (the purified PHB). C) Prohibitin identified by mass spectrometry 
galactopyranoside (IPTG), the recombination cells were lysed ultrasonically and the cell extracts were analysed by SDS-PAGE. High levels of recombinant prohibitin were observed (Fig. 1B). The protein was analysed by means of LC-MALDI-TOF/TOF (Fig. 1C).

\section{Western blotting detection}

Western blotting was performed to detect the autoantibodies in patients' sera of RA. From the results, bound antibodies against the $30-\mathrm{kDa}$ band were observed in three out of eight sera of RA, suggesting prohibitin may be a target antigen of RA. But no positive reaction was observed in healthy controls (Fig. 2A).

\section{Immunoprecipitation validation}

Immunoprecipitation was further performed between rhPHB protein and RA sera. The results show that the band of rhPHB protein was clearly present in the immunoprecipitates, further indicating that prohibitin was a target antigen of RA (Fig. 2B). The protein band was excised and identified as prohibitin by mass spectrometry (Fig. 2C).

\section{ELISAs}

The presence of anti-prohibitin antibodies was detected by ELISA. The results showed the frequency of antibodies to prohibitin in sera from different types of diseases. Of the 86 sera with RA tested, 26 (30.3\%) were found to be anti-prohibitin positive, and 7 of 86 SLE patients (8.1\%) and $1(1.2 \%)$ of 86 healthy controls were observed as positive. Statistical analysis indicated that there was a significant difference $(* * * p<0.0001)$ in anti-prohibitin antibody production between RA and HC (Fig. 3).

\section{Discussion}

By using western blotting and immunoprecipitation we observed that the rhPHB protein was successfully recognised by serum IgG from RA, suggesting prohibitin as a putative target autoantigen in RA. Moreover, the result of positive incidence of anti-prohibitin antibodies between western blotting and ELISA is consistent.

Rheumatoid arthritis is mainly characteristic of cellular immunity dysfunction, particularly the imbalances of Th1 and Th2 cells in the CD4-positive T cell, resulting from $\mathrm{T}$ and $\mathrm{B}$ lymphocyte activation that participates in the immunological effect and produces lots of cytokines, and high levels of immunoglobulin and autoantibodies, resulting in joint synovial inflammation [26-28]. Terashima et al. [17] reported the association of prohibitin and the antigen receptor on B lymphocytes and speculated that prohibitin can inhibit the proliferation of lymphocytes. Also, prohibitin has the function of inhibiting cellular proliferation, so the existence of serum anti-prohibitin antibodies may cause corresponding function dis-

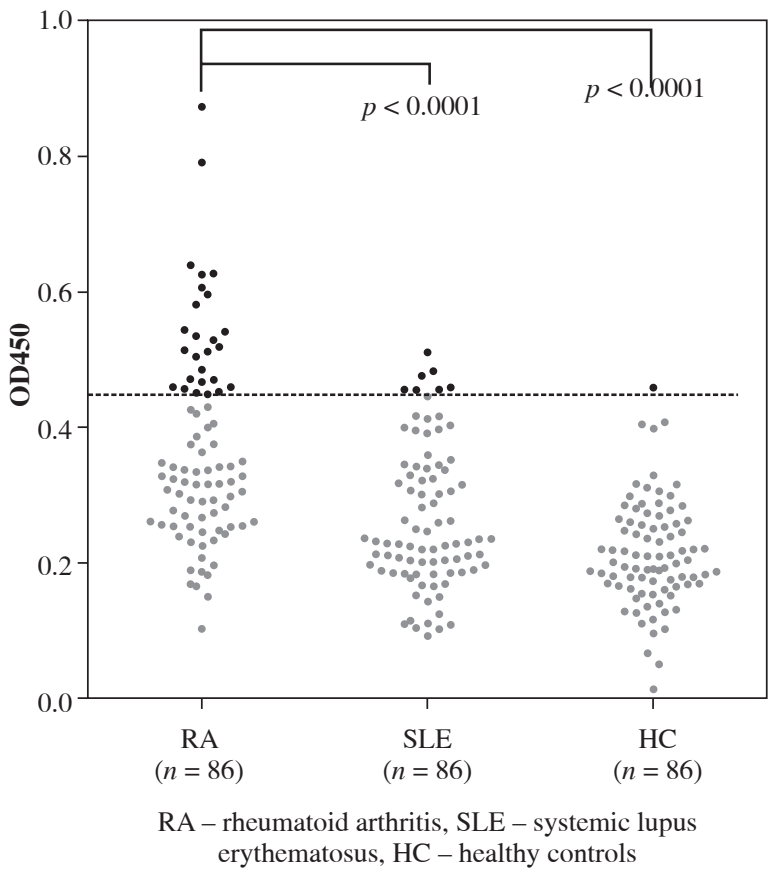

Fig. 3. The prevalence of anti-prohibitin antibodies in sera from patients. ELISA was used to detect the reactivity of serum IgG against recombinant human prohibitin protein. The anti-prohibitin antibodies were detected in 26 of 86 RA patients $(30.3 \%), 7$ of 86 SLE patients $(8.1 \%)$, and 1 of 86 healthy donors $(1.2 \%)$. The reactivity of anti-PHB antibodies in RA was significantly higher than $\operatorname{SLE}(* * * p<0.0001)$ and $\mathrm{HC}(* * * p<0.0001)$

order, eventually leading to inflammation. Continuous expression of prohibitin in intestinal epithelial cells can inhibit TNF- $\alpha$-induced NF- $\kappa \mathrm{B}$ activation [20], and this interaction may contribute to improving the understanding of RA.

In summary, this study revealed prohibitin is a putative autoantigen in RA, and the corresponding autoantibody was present in about one third of patients' blood circulation.

The authors declare no conflict of interest.

The authors wish to express their gratitude for the help of Prof. Dr. Yaping Tian and Dr. Chunyan Zhang from the Chinese PLA General Hospital. And the same gratitude is expressed to Prof. Dr. Shutao Sun from the Institute of Microbiology, Chinese Academy of Sciences for MS analysis. This work was supported by Fundamental Research Funds for the the Central Universities, Program for New Century Excellent Talents in University and the National Natural Science Foundation of China (No. 31371203). 


\section{References}

1. Alamanos Y, Drosos A (2005): Epidemiology of adult rheumatoid arthritis. Autoimmun Rev 4: 130-136.

2. Kourilovitch M, Galarza-Maldonado C, Ortiz-Prado E (2014): Diagnosis and classification of rheumatoid arthritis. J Autoimmun 48-49: 26-30.

3. McInnes IB, Schett G (2011): The pathogenesis of rheumatoid arthritis. N Engl J Med 365: 2205-2219.

4. van Venrooij WJ, Pruijn GJ (2014): How citrullination invaded rheumatoid arthritis research. Arthritis Res Ther 16: 103.

5. Mageed RA (1996): The RF antigen. In: Manual of Biological Markers of Disease. van Venrooij WJ, Maini RN (eds.). Springer Press, Dordrecht 1996; 167-193.

6. Masson-Bessičre C, Sebbag M, Girbal-Neuhauser E, et al. (2001) The major synovial targets of the rheumatoid arthritis-specific antifilaggrin autoantibodies are deiminated forms of the alpha- and beta-chains of fibrin. J Immunol 166: 4177-4184.

7. Vossenaar ER, Després N, Lapointe E, et al. (2004): Rheumatoid arthritis specific anti-Sa antibodies target citrullinated vimentin. Arthritis Res Ther 6: R142-R150.

8. Kinloch A, Tatzer V, Wait R, et al. (2005): Identification of citrullinated $\alpha$-enolase as a candidate autoantigen in rheumatoid arthritis. Arthritis Res Ther 7: R1421-1429.

9. Kim W, Cho M, Jung YO, et al. (2004): Type II collagen autoimmunity in rheumatoid arthritis. Am J Med Sci 327: 202-211.

10. Iwaki-Egawa S, Matsuno H, Yudoh K, et al. (2004): High diagnostic value of anticalpastatin autoantibodies in rheumatoid arthritis detected by ELISA using human erythrocyte calpastatin as antigen. J Rheumatol 31: 17-22.

11. Okazaki Y, Suzuki A, Sawada T, et al. (2006): Identification of citrullinated eukaryotic translation initiation factor $4 \mathrm{G} 1$ as novel autoantigen in rheumatoid arthritis. Biochem Biophys Res Commun 341: 94-100.

12. Sekine T, Masuko-Hongo K, Matsui T, et al. (2001): Recognition of YKL-39, a human cartilage related protein, as a target antigen in patients with rheumatoid arthritis. Ann Rheum Dis 60: 49-54.

13. Wegner N, Lundberg K, Kinloch A, et al. (2010): Autoimmunity to specific citrullinated proteins gives the first clues to the etiology of rheumatoid arthritis. Immunol Rev 233: 34-54.

14. Bax M, Huizinga TW, Toes RE (2014): The pathogenic potential of autoreactive antibodies in rheumatoid arthritis. Semin Immunopathol 36: 1-13.

15. Theiss AL, Sitaraman SV (2011): The role and therapeutic potential of prohibitin in disease. Biochim Biophys Acta 1813: 1137-1143.

16. Theiss AL, Idell RD, Srinivasan S, et al. (2007): Prohibitin protects against oxidative stress in intestinal epithelial cells. FASEB J 21: 197-206.

17. Mishra S, Murphy LC, Nyomba BL, Murphy LJ (2005): Prohibitin: a potential target for new therapeutics. Trends Mol Med 11: 192-197.

18. Kishimoto T (2005): Interleukin-6: from basic science to medicine-40 years in immunology. Annu Rev Immunol 23: 1-21.

19. Brennan FM, Maini RN, Feldmann M (1992): TNF $\alpha-$ a pivotal role in rheumatoid arthritis? Rheumatology 31: 293-298.

20. Theiss AL, Jenkins AK, Okoro NI, et al. (2009): Prohibitin inhibits tumor necrosis factor alpha-induced nuclear factor-kappa B nuclear translocation via the novel mechanism of decreasing importin $\alpha 3$ expression. Mol Biol Cell 20: 4412-4423.
21. Xun Y, Chen P, Yan H, et al. (2014): Identification of prohibitin as antigen in Behcet's disease. Biochem Biophys Res Commun 451: 389-393.

22. Arnett FC, Edworthy SM, Bloch DA, et al. (1988): The American Rheumatism Association 1987 revised criteria for the classification of rheumatoid arthritis. Arthritis Rheum 31: 315-324.

23. Tan EM, Cohen AS, Fries JF, et al. (1982): The 1982 revised criteria for the classification of systemic lupus erythematosus. Arthritis Rheum 25: 1271-1277.

24. Du H, Chen G, Wang S, et al. (2012): Immunological screening and characterization of highly specific monoclonal antibodies against $20 \mathrm{kDa}$ hGH. Bioanalysis 4: 2161-2168.

25. Du H, Li C, Jin H, et al. (2013): Generation and evaluation of antibodies against human MGF E-peptide by reverse phase protein microarray and reverse competitive ELISA. Bioanalysis 5: 2269-2275.

26. Epstein FH, Choy EH, Panayi GS (2001): Cytokine pathways and joint inflammation in rheumatoid arthritis. N Engl J Med 344: 907-916.

27. Korganow A, Ji H, Mangialaio S, et al. (1999): From systemic $\mathrm{T}$ cell self-reactivity to organ-specific autoimmune disease via immunoglobulins. Immunity 10: 451-461.

28. Skapenko A, Leipe J, Lipsky PE, Schulze-Koops H (2005): The role of the $\mathrm{T}$ cell in autoimmune inflammation. Arthritis Res Ther 7: S4-14. 